\title{
Low-cost Sensor Tape for Environmental Sensing Based on Roll-to-roll Manufacturing Process
}

\author{
Nan-Wei Gong ${ }^{1}$, Chiu-Yen Wang ${ }^{2}$, Joseph A. Paradiso ${ }^{1}$ \\ ${ }^{1}$ Responsive Environments Group, MIT Media Lab, Cambridge MA, USA \\ ${ }^{2}$ Department of Material Science and Engineering, National Tsing Hua University, Taiwan
}

\begin{abstract}
We describe the concept of fabricating low-cost sensor tape for fine-grid environmental sensing based on roll-toroll manufacturing processes. We experiment with constructing sensors and electronic connections with low-cost conductive inkjet printed copper traces. Our first attempt is to fabricate humidity sensors by spin-coating conductive polymer on sensor substrates and integrating the design with an embedded system. The humidity sensor was tested in a two-point probe and exhibits the I-V profile of a diode. We demonstrated a working humidity sensor with an impedance variance of $30 \mathrm{k} \Omega$ from $99 \%$ to $58 \%$ RH within 300 seconds under a 1.4 volt bias.
\end{abstract}

\section{INTRODUCTION}

Design and integration of fine grid environmental sensing has always been a major challenge. Deploying a dense sensor network nowadays involves implementing individual sensors and complex wiring between the processor and sensors. The cost of individual sensor manufacturing and system integration is high; hence, in most cases, engineers use interpolation to compensate the missing gaps. Accordingly, we envision a low-cost, large-coverage sensing tape based on roll-to-roll manufacturing where the substrate, electronic circuitry, electrode, and sensing elements are implemented in the same process. Different sensing elements can be realized, e.g., either electromagnetic field sensing components that are embedded in the copper trace design or chemical sensing that is based on coating resistive polymers on top of the copper trace "template" for multiple sensing targets.

This one-step fabrication process opens up a possibility for inexpensive dense sensor system deployment that is unlimited in length and also different applications for large-area environmental sensing. In order to have the flexibility of grid compactness for both narrow and wide area coverage, we decided to experiment with a "tape" structured linear geometry with dense sensing capabilities. This shape enables the user to physically manipulate and configure the sensing density for various measurements. The sensory units can be more precisely placed in a targeted location, like how the density and receptors' functionalities on our skin vary based on the location of the skin.

We started our experiment with conductive inkjet technology [1], a process that enables us to print copper on Mylar (polyester) through a roll-to-roll printing and plating process. Our previous work includes designing electromagnetic field sensors on the substrate for location tracking, gait analysis, RF communication [2] and customizable surfaces for music control [3]. The surface is scalable, flexible, and flat for easy adaptation in the implementation of a large space. However, the application is limited by the sensing modalities. Here, we present the possibility of adding more sensing elements by adding a layer of conductive polymer, which can potentially be integrated into the same roll-to-roll manufacturing process as the circuitry printing process. In this paper, we show one example of the integration of printed sensor tape with humidity sensing capability.

\section{BACKGROUND}

\section{A. Sensate Surcfaces}

Our inspiration for developing flexible sensor systems comes from the conformity of human skin. Our skin, which covers our whole bodies, is the direct interface between us and the physical world. It not only serves as the first line of protection, it also incorporates various sensing modalities densely into the substrate. With compact distribution of special sensory receptors, skin is capable of fine spatial resolution and high sensitivity. These capabilities point toward the development of a dense, multi-modal, flexible, distributed sensate "electronic skin" platform - a fine-grained interface between digital and physical worlds inspired by human skin.

Most platforms for constructing electric skin are designed for robotics (tactile presence and proximity sensing to avoid collision), telemedicine (tactile feedback for remote examination or even surgery) and other systems that require lightweight, compact, thin layers of sensory output. Like real human skin, electric skin should be soft, stretchable, and exhibit ubiquitous surface coverage. The constraint of traditional electronic fabrication techniques that produce planar surfaces (printed circuit boards) has been a great barrier for researchers to implement these ideas. Recent developments in materials and mechanics for stretchable electronics [4-6] such as chemically-constructed microstructures for embedding sensing matrices in composite films, provide a promising future for electric skin; however, those technologies are still far away from full-scale mass production.

Many researchers have developed dense, skin-like sensor systems. The benefit and technological requirements of having sensitive skin devices were first demystified by Lumelsky et 
al. [7-8], who envisioned a new paradigm in sensing and control enabled by large arrays of sensors embedded in a flexible, stretchable, and/or foldable substrate that could cover the surface of a moving machine. These surfaces can aid unsupervised machinery in unpredictable and unstructured surroundings. Most of the subsequent research focused on robotic tactile sensing [7-8], capacitive matrices for HumanComputer Interaction [9-12], and related research in distributed sensor networks [13-14]. Strategies of peer-to-peer communication in computer algorithms for dense sensor network platforms were developed to process the massive amount of information generated via a skin interfaces [13-14].

Another major area of research in this area is the development of materials and manufacturing methods for electronic skins. Scientists seek to embed nano-scaled active matrix arrays of transducers using flexible materials [15-16]. This area is closely related to the development of flexible electronics that is covered in the following section. Here, we list several examples of state-of-the-art research that is directly linked to the development of sensate skin interfaces. Wagner et al. [15] proposed the architecture and components necessary for electronic skin: elastomeric skin that carries rigid islands on which active sub-circuits are made. Some of the technologies are now almost ready for commercialized usage they enable electronics to bend, stretch and wrap into novel form factors [16] by exploiting techniques used in the existing semiconductor industry infrastructure.

Several recent research results showed a promising future in fabricating flexible arrays of transducers with pressure sensitivity and response times approaching those of natural human skin [17-18]. These examples demonstrated a promising future for skin-type sensing interfaces and an immediate need for developing computing algorithms and applications for the processing, analysis and use of data generated by these platforms.

\section{B. Flexible, Stretchable Electronics}

Flexible electronics has gained significant interest in the manufacturing of circuits due to the potential of alternative printed circuit board fabrication techniques such as roll-to-roll processes, which could lead to low-cost, high manufacturing speed, large-area electronics.

There are three major types of possible fabrication techniques for flexible electronics that can be applied to the manufacturing process of a sensing skin. The first one is fabrication on web by roll-to-roll processing [19]. Roll-to-roll lithography tools are capable of micron resolution on flexible substrate materials. In roll-to-roll processing, the device layers can be patterned by additive processes of directly printing the active materials, shadow masking, or subtractive patterning by photolithography [19]. Another manufacturing method for fabricating large area, low cost skin structures is additive printing. This process is roll-to-roll compatible. For most industrial applications of flexible electronics, the focus is on printing TFT or capacitive touch screens. This process can print noble-metal conductors, organic conductors, semiconductors and even insulators. Most researchers are now studying different synthesis methods for printing metallic conductors from nanoparticles [21]. Printing from

nanoparticles could reduce the required sintering temperature to values that are acceptable for plastic substrates. Meanwhile, we can get more flexibility by using a polymer as the printing substrate. Among all the mass production factories, there are a few currently accessible methods for end users to experiment on such as Copper-on-Kapton substrates, Conductive Inkjet Flex technology [1], and metallic nanoparticle inkjet printing [20]. In this paper, we choose Conductive Inkjet Flex for our sensor skin construction.

\section{METHOD AND EXPERIMENT}

We have begun to add more sensing capabilities by coating polyimide films on our flexible electronics circuit layouts. Polyimide film has a high water absorption property because of its imide bonds, which causes changes in space charge density under different humidity conditions. Also, it is stable and hence requires no extra packaging. We adapted spin-coating as our method for testing different conditions. Our material selection focused on finding a low-temperature curable polyimide $\left(\sim 150 \mathrm{C}^{\circ}\right)$ to match the melting point of our flexible electronics substrate, Polyester. In our experiment, we used positive tone photosensitive polyimide, Photoneece $\mathbb{B}$ (PW-1500), which is commonly used for buffer and insulation layers in semiconductors and electronic components as a humidity sensing material.

(a)

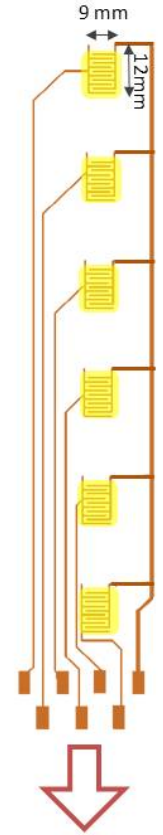

Low-temperature curable polyimide on copper electrode

Copper electrode on polyester substrate
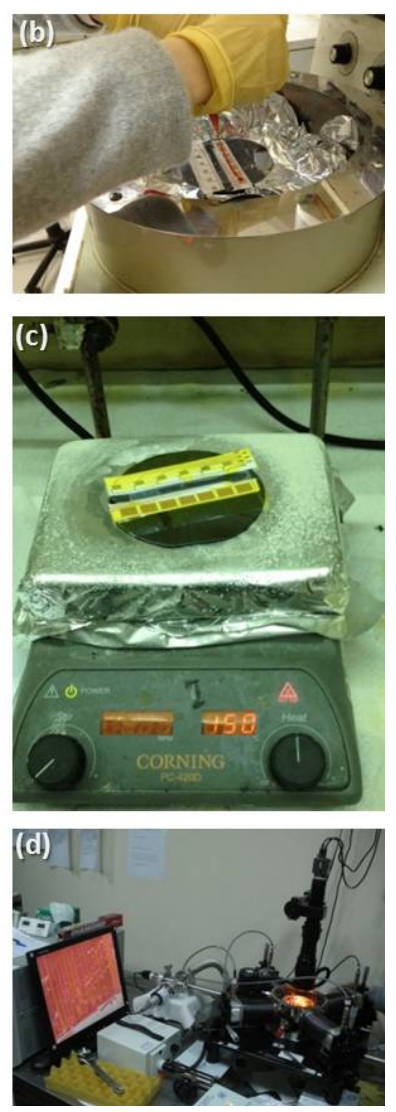

Figure 1. (a) Sensor design - copper electrode on polyester substrate coated with low-temperature curable polyimide. (b) Spin coating $20 \mu \mathrm{m}$ thick polyimide onto conductive inkjet-printed substrate. (c) Pre-bake and curing on a hot plate. (d) IV sweep measurements with 2 point probe. 
We experimented using $9 \mathrm{~mm} * 12 \mathrm{~mm}$ film with $20 \mu \mathrm{m}$ thickness (figure 1(a)) of polyimide coating. The film was produced with spin coating.

The polyimide material was first applied on top of the flexible electronics substrate and then spin-coated at $700 \mathrm{rpm}$ for 10 seconds and then $2750 \mathrm{rpm}$ for 30 seconds (Figure 1(bc)). The samples were then prebaked at $120^{\circ} \mathrm{C}$ for 3 minutes on a hot plate. The last step was to cure the polymer on the hot plate at $150{ }^{\circ} \mathrm{C}$ for 180 minutes. The I-V curve was measured with a two-point probe system.

\section{RESULTS AND DISCUSSIONS}

The I-V curve for all samples was consistent with a forward bias for the $0.8 \mathrm{~cm}^{2}, 20 \mu \mathrm{m}$ thick polyimide film under $58 \% \mathrm{RH}$, as shown in figure 2 .

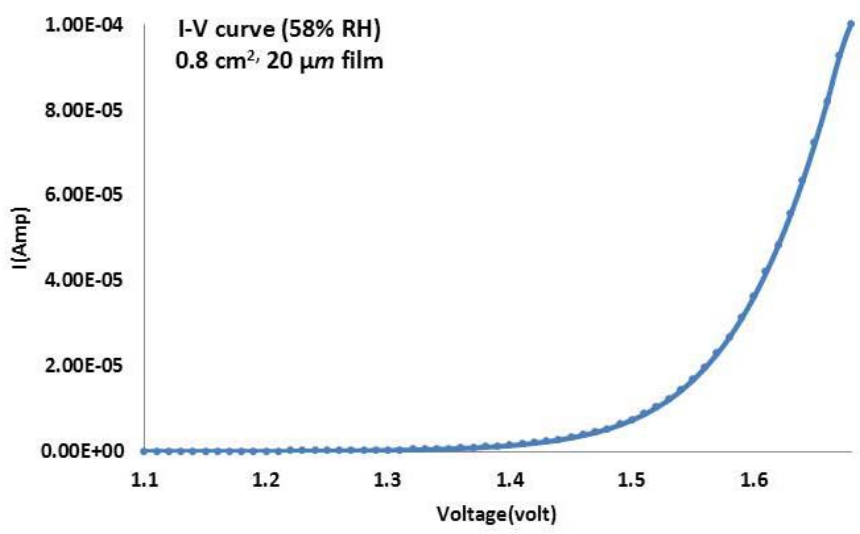

Figure 2. $\mathrm{I}-\mathrm{V}$ curve of a $0.8 \mathrm{~cm}^{2}, 20 \mu \mathrm{m}$ thick film at $58 \%$ relative humidity.

Since the I-V curve was consistent with a forward bias, to test the sensitivity of this material with humidity, we designed a current-to-voltage converter circuit to measure and amplify the signal. Figure 3(a) is our circuit design - the operational amplifier (TLV2371) provides a constant voltage for the humidity sensor, while $R_{1}$ in the negative feedback loop amplifies the signal by $\mathrm{R}_{1} / \mathrm{R}_{\text {sensor }}$ times.

Figure 3(b) is the picture of an example circuit implementation. The four output leads were connected to the analog-to-digital input of a microcontroller. The main concern about having a long cascaded line of sensing elements is the line impedance and signal drop. In our implementation, we measured $6 \Omega$ of impedance caused by the copper traces in a 0.3 meter line, which could cause less than $1 \%$ of variation in the sensor reading. With multiplexing at the end of each strip of sensor tape, we could read the humidity measurement of each sensing element with minimum components attached to one reel of tape.

To test the response time of our humidity sensing element, we started by placing our sensor under $99 \% \mathrm{RH}$, then back to $58 \%$ RH, which was the ambient humidity. Figure 4 shows an impedance variation of $30 \mathrm{k} \Omega$ from $99 \%$ to $58 \% \mathrm{RH}$ within 300 seconds under constant bias of 1.4 volts. Although the response time was not as fast as most polymer-based capacitive humidity sensors [22], the value is repeatable and responsive to humidity changes. Response time could be improved by increasing film thickness or using polymer with higher water absorption ability (for PW-1500, the water absorption rate is $4 \%$ ).
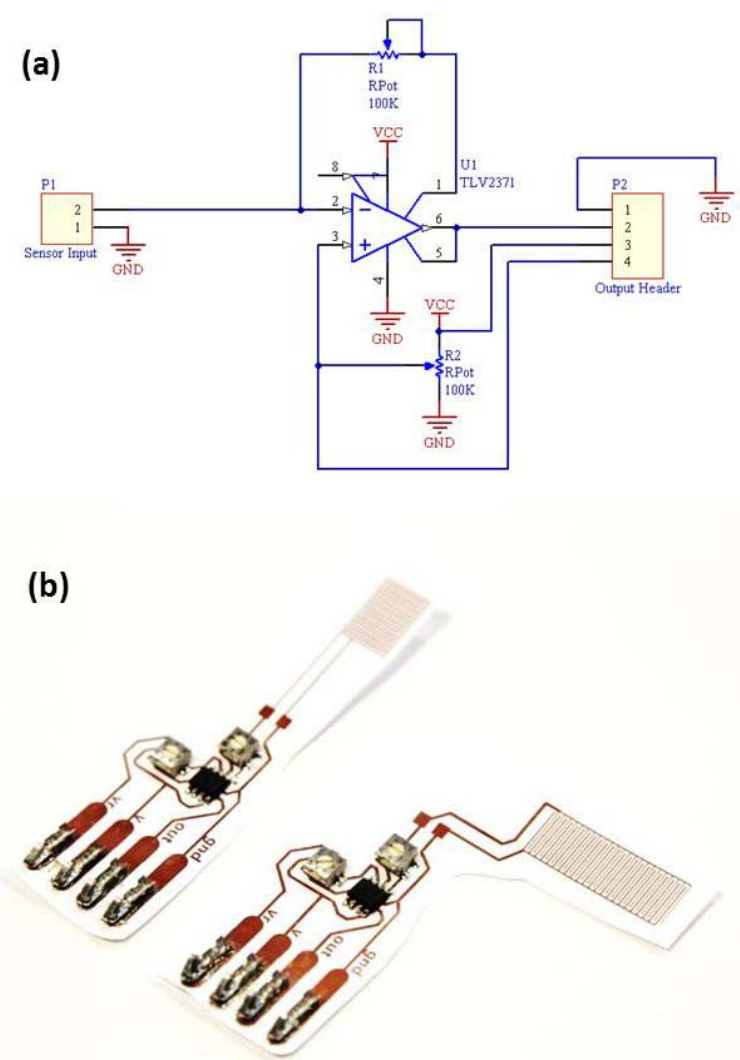

Figure 3. (a) Circuit diagram for measuring resistance change at a fixed voltage. (b) Picture of the actual circuit and components. The four pins are connected to power, ground, reference voltage and amplifier voltage output linked to the analog-to-digital input of a micro-processor.

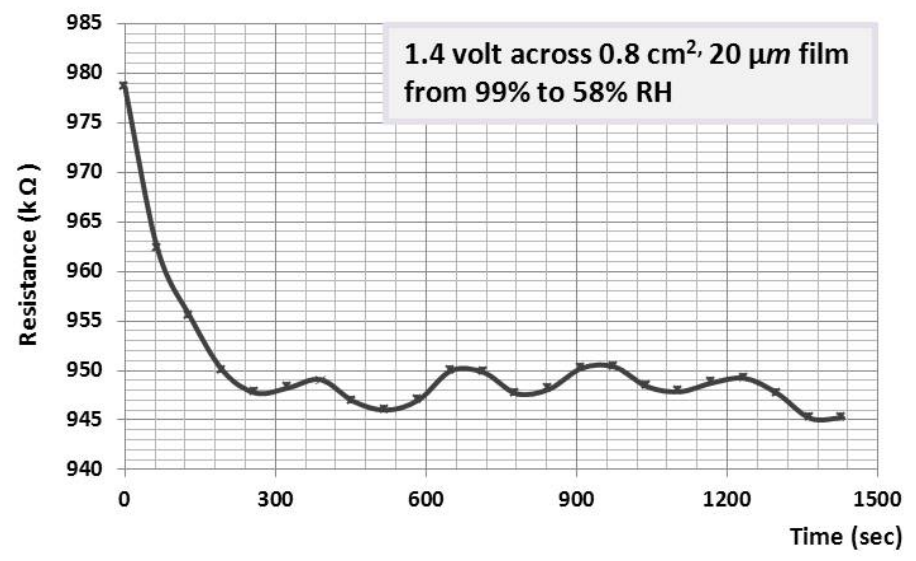

Figure 4. Impedance variance of $30 \mathrm{k} \Omega$ from $99 \%$ to $58 \% \mathrm{RH}$ within 300 seconds under 1.4 volts. 


\section{CONCLUSION}

In this paper, we described the concept and provided an example of fabricating low-cost humidity sensing tape for fine-grid environmental sensing based on roll-to-roll manufacturing process. We experimented with the possibility of constructing sensors and sensor templates with a low-cost conductive inkjet printing copper circuitry.

Our first attempt was to fabricate humidity sensors by spin-coating low-temperature curable, high water absorption, conductive polymer (polyimide) on to our sensor substrate and integrate the design with an embedded system for data collection and power management. The humidity sensor was tested in a two-point probe and exhibits the I-V profile of a diode. We utilized this property and designed a current-tovoltage converter circuit to measure and amplify the signal. We demonstrate a working humidity sensor prototype with impedance varying by $30 \mathrm{k} \Omega$ from $99 \%$ to $58 \%$ relative humidity (RH) within 300 seconds under 1.4 volts.

Future work includes developing multiple sensing modalities such as temperature and light on the same template for the production a linear sensor tape with an assortment of sensing abilities. We are also exploring methods of wirelessly powering the tape so it can be remotely interrogated without needing batteries or direct data [23]. We envision a flexible, inexpensive, low power sensor tape system that can be embedded into construction materials as a building block of smart structures in the future.

\section{ACKNOWLEDGMENT}

The authors acknowledge the help and support of members of the Responsive Environments Group at the MIT Media Lab and the Nano Structures and Dynamics Lab at National Tsing Hua University.

\section{REFERENCES}

[1] http://www.conductiveinkjet.com/

[2] N.-W. Gong, S. Hodges and J. A. Paradiso," Leveraging Conductive InkjetTechnology to Build a Scalable and Versatile Surface for Ubiquitous Sensing" InProc. of the 13th international conference on Ubiquitous computing (UbiComp '11), 45-54.

[3] N.-W. Gong, N. Zhao and J. A. Paradiso," A Customizable Sensate Surface for Music Control," In Proc. of the 12th international conference on New Interfaces for Musical Expression (NIME 12), pp. 417-420.

[4] K. S. Kim et al, Large-scale pattern growth of graphene films for stretchable transparent electrodes. Nature 457 (7230), 706-10, 14 Jan 2009 .
[5] T. Sekitani et al., A Rubberlike Stretchable Active Matrix Using Elastic Conductors. Science 321 (5895), 12 Sep 2008.

[6] J. Rogers, T. Someya, and Y. Huang, "Materials and Mechanics for Stretchable Electronics," Science 327 (5973), 26 Mar 2010.

[7] V. J. Lumelsky, M. S. Shur and S. Wagner, "Sensitive Skin," IEEE Sensors J, 1, No 1, June 2001, pp 41-51.

[8] T. Papakostas, J. Lima and M. Lowe, "Large area force sensor for smart skin applications," Proceedings of the IEEE Sensors 2002 Conference, 2, 1614-1619, 2002.

[9] W. D. Stiehl et at., "The huggable: A therapeutic robotic companion for relational, affective touch," IEEE CCNC 2006 Proceedings, 2006.

[10] D. Richardson, "Smart skins and structures," Armada International, 22, No 2, pp 50-56, April/May 1998.

[11] P. Dietz and D. Leigh, "DiamondTouch: a multi-user touchtechnology," in Proc of ACM UIST 2001, Orlando, Florida, USA, pp 219-226, Nov 2001.

[12] B. Richardson, K. Leydon, M. Fernström, J. Paradiso, "Z-Tiles: building blocks for modular, pressure-sensing floor spaces," In: Extended Abstracts of the 2004 conference on Human factors and computing systems, Vienna, Austria, pp. 1529-1532, 2004.

[13] J. A. Paradiso, J. Lifton, and M. Broxton, "Sensate Media - Multimodal Electronic Skins as Dense Sensor Networks," BT Technology Journal, Vol. 22, No. 4, October 2004, pp. 32-44.

[14] J. Lifton, M. Broxton, J.A. Paradiso, "Distributed sensor networks as sensate skin," In: Proceedings of the 2nd IEEE international conference on sensors (Sensors 2003), Toronto, Canada, October 2003, pp 743747.

[15] S. Wagner et al., "Electronic skin: architecture and components," Physica E: Low-dimensional Systems and Nano structures Volume 25, Issues 2-3, November 2004, pp 326-334.

[16] www.mc10.com

[17] K. Takei et al., "Nanowire active-matrix circuitry for low-voltage macroscale artificial skin," Nature Materials 9 (10), 821-6, 20 Oct 2010.

[18] S. C. B. Mannsfeld et al., "Highly sensitive flexible pressure sensors with microstructured rubber dielectric layer," Nature Materials doi:10.1038/nmat2834, 12 Sep 2010.

[19] W. S. Wong and A. Salleo, "Flexible Electronics: Materials and Applications", 2009.

[20] B. Walker and J. A. Lewis, "Reactive Silver Inks for Patterning HighConductivity Features at Mild Temperatures," S. Journal of the American Chemical Society 2012134 (3), pp 1419-1421.

[21] S. B. Fuller, E.J. Wilhelm, J.M. Jacobson, J. Microelectromechanical Syst. 11 (2002) 54.

[22] H. Grange, C. Bieth, H. Boucher, and G. Delapierre, "A capacitive humidity sensor with very fast response time and very low hysteresis," Sens. \& Actuators, 1987,12, pp. 291-296.

[23] R.Vyas, V. Lakafosis, M. M.Tentzeris, H. Nishimoto and Y. Kawahara, "A Battery-less Wireless Mote for Scavenging Wireless Power at UHF (470-570 MHz) Frequencies", Procs. Of the 2011 IEEE Antenna and Propagation Symposium, Spokane, WA, July 2011. 